\title{
Response to the Letter to the Editor by Waarsenburg et al.
}

\author{
Farah Lone $^{1} \cdot$ Ranee Thakar ${ }^{1} \cdot$ Abdul H. Sultan ${ }^{1}$
}

Published online: 4 August 2015

(C) The International Urogynecological Association 2015

\section{Dear Editor,}

We thank Kim van de Waarsenburg et al. for their interest in our paper [1].

Unfortunately, they have misread our paper, as this was not a randomised trial but an observational prospective study and therefore was not analysed according to intention to treat. All women with symptomatic pelvic organ prolapse (POP) referred to a specialist urogynaecology clinic were offered a choice of pessary or surgery as their first-line treatment.

Women who were excluded because they discontinued pessary use were not necessarily dissatisfied with its use, and the reasons for discontinuation - already in the Results sectionare as follows: difficulty retaining the pessary (7), vaginal discomfort (2) and vaginal discharge (3). Although eight women $(6 \%)$ did not experience any untoward symptoms, they changed their decision and opted for surgery and were excluded from the analysis.

The 1-year follow-up achieved in our study is better and certainly comparable with most prospective questionnaire follow-up studies [2-6]. This cannot be referred to as "selective loss to follow-up" but represents the real-life situation when women are treated with pessaries or surgery. Moreover, in both groups, almost similar numbers did not complete the 1-year questionnaire (53 and 51, respectively), as shown in Fig. 2 [1].

As pelvic floor dysfunction symptoms are best described in accordance with degree of bother, we used the validated ICIQ questionnaire, which assesses bothersomeness of symptoms

This reply refers to the comment available at doi:10.1007/s00192-015-2812-8.

Ranee Thakar

ranee.thakar@croydonhealth.nhs.uk

1 Department of Obstetrics and Gynecology, Croydon University Hospital, 530 London Road, Croydon, Surrey CR7 7YE, UK
$[7,8]$. Our study was not designed to enable interpretation of symptoms on an individual basis; perhaps this is best answered in a qualitative study. Also, the study was designed to assess the cost effectiveness of a primary treatment strategy.

To our knowledge, this is the first prospective study to evaluate comparative outcomes of vaginal pessaries and surgery using the validated ICIQ-VS and ICIQ-UI (SF) questionnaires at 1 year. At this time, the study provides the best available evidence base by which to counsel women.

\section{References}

1. Lone F, Thakar R, Sultan AH (2015) One-year prospective comparison of vaginal pessaries and surgery for pelvic organ prolapse using the validated ICIQ-VS and ICIQ-UI (SF) questionnaires. Int Urogynecol J. doi:10.1007/s00192-015-2686-9

2. Kapoor DS, Thakar R, Sultan AH, Oliver R (2009) Conservative versus surgical management of prolapse: what dictates patient choice? Int Urogynecol J 20(10):1157-1161

3. Bradshaw HD, Hiller L, Farkas AG, Radley S, Radley SC (2006) Development and psychometric testing of a symptom index for pelvic organ prolapse. J Obstet Gynaecol 26:241-252

4. Barber MD, Walters MD, Cundiff GW, PESSRI Trial Group (2006) Responsiveness of the pelvic floor distress inventory (PFDI) and pelvic floor impact questionnaire (PFIQ) in women undergoing vaginal surgery and pessary treatment for pelvic organ prolapse. Am J Obstet Gynecol 194(5):1492-1498

5. Kelleher CJ, Cardozo LD, Khullar V, Salvatore S (1997) King's health questionnaire (KHQ). a new questionnaire to assess the quality of life of urinary incontinent women. BJOG 104:1374-1379

6. Schaffer J, Cundiff GW, Amundsen CL, Bent A, Coates KW, Strohbehn K et al (2006) Do pessaries improve lower urinary tract symptoms? J Pelvic Med Surg 12:72-73

7. Price N, Jackson SR, Avery K, Brookes ST, Abrams P (2006) Development and psychometric evaluation of the ICIQ vaginal symptoms questionnaire: the ICIQ-VS. BJOG 113(6):700-712

8. Avery K, Donovan J, Peters T, Shaw C, Gotoh M, Abrams P (2004) ICIQ: a brief and robust measure for evaluating the symptoms and impact of urinary incontinence. Neurourol Urodyn 23(4):322-330 